Hautarzt 2009 - 60:284-284

DOI 10.1007/s00105-009-1752-y

Online publiziert: 26. März 2009

(c) Springer Medizin Verlag 2009

\section{J. Krutmann}

Institut für Umweltmedizinische Forschung, Heinrich-

Heine-Universität Düsseldorf gGmbH

\title{
Moderner Lichtschutz in der ästhetischen Dermatologie
}

Das zentrale Anliegen der ästhetischen Dermatologie ist es, den Hautalterungsprozess der steigenden Lebenserwartung anzugleichen. Hautalterung wiederum ist die Konsequenz von intrinsischen und extrinsischen Prozessen, wobei Letztere durch eine Vielzahl von Strategien beeinflussbar sind. An erster Stelle zu nennen ist hier die konsequente Prävention der extrinsischen Hautalterung durch einen wirksamen Lichtschutz. Das vorliegende Leitthemenheft gibt einen aktuellen Überblick über die Möglichkeiten, aber auch neu erkannte Herausforderungen, potenzielle Risiken und Grenzen des modernen Lichtschutzes.

\section{(7) Der konventionelle UV-Schutz gegen UVA- und UVB-Strahlung hat ein hervorragendes Leistungsniveau erreicht}

So wurde in den letzten Jahren die Entwicklung neuer Breitband-UV-Filter, die nicht nur öl-, sondern auch wasserlöslich sind und die durch geschickte Kombination additive und synergistische Effekte erzielen können, beispielhafterweise vorangetrieben mit dem Ergebnis, dass der konventionelle, d. h. auf Filtern basierende UV-Schutz gegen UVA- und UVB-Strahlung außerordentlich wirksam ist.

Flankiert wird diese Entwicklung durch einen zweiten Prozess, der in den nächsten Jahren stetig an Bedeutung gewinnen wird, nämlich die Entwicklung von biolo- gisch aktiven Substanzen, die nicht passiv durch eine Filterwirkung, sondern aktiv durch den Eingriff in Signalabläufe oder die Stimulation von Reparatur- bzw. Regenerationsprozessen zum Schutz der Haut vor Sonnenstrahlung beitragen.

Diesen positiven Entwicklungen steht jedoch auch die Erkenntnis gegenüber, dass nicht nur UVA- und UVB-Strahlung, sondern auch andere Spektralbereiche innerhalb der Sonnenstrahlung zur Schädigung der Haut und insbesondere zu einem vorzeitigen Hautalterungsprozess beitragen. An erster Stelle ist hier die kurzwellige Infrarotstrahlung (IRA) zu nennen. Neueste Erkenntnisse zur IR-A-induzierten Signaltransduktion in menschlichen Hautzellen weisen darauf hin, dass grundsätzlich ein IR-A-Schutz möglich ist und daher Bestandteil eines umfassenden, d. h. wirksamen Sonnenschutzes werden wird.

Bei aller Euphorie über den präventivmedizinischen Fortschritt, der in den letzten Jahren bei der Verhinderung extrinsischer Hautalterungsprozesse erzielt wurde, darf nicht vergessen werden, dass neue Technologien auch neue Risiken bergen können. Deutlich wird dies an der aktuellen Diskussion über die Toxizität von Nanopigmenten. So stellen Nanopartikel aufgrund ihrer ubiquitären Verbreitung in modernen Industriegesellschaften eines der größten zukünftigen umweltmedizinischen Risiken für den Menschen dar, und neuere Arbeiten deuten darauf hin, dass diese weniger als $100 \mathrm{~nm}$ großen Partikel auch durch die Hautbarriere zu penetrieren vermögen.

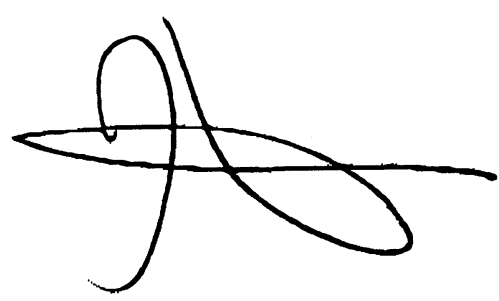

Ihr Jean Krutmann

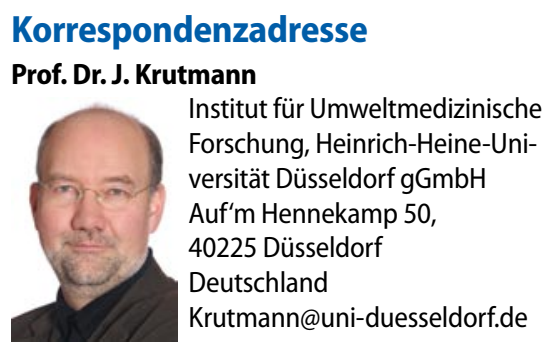

